\title{
Endoscopic submucosal dissection for early gastric cancer without interruption of warfarin and aspirin
}

Authors

Institutions
Shigetaka Tounou' ${ }^{1}$, Yasushi Morita², Tomohiro Hosono², Hideaki Harada², Kenji Hayasaka², Yasushi Katsuyama², Satoshi Suehiro ${ }^{2}$, Seishi Nagano ${ }^{2}$, Takanori Shimizu²

${ }^{1}$ Teikyo University Chiba Medical Center - Gastroenterology, Ichihara, Chiba, Japan

${ }^{2}$ Shin-Tokyo Hospital - Gastroenterology, Matsudo, Chiba, Japan submitted

28. September 2014 accepted after revision 5. March 2015

\section{Bibliography}

DOI http://dx.doi.org/

10.1055/s-0034-1392018

Published online: 12.6.2015

Endosc Int Open 2015; 03:

E307-E310

(c) Georg Thieme Verlag KG

Stuttgart · New York

E-ISSN 2196-9736

\section{Corresponding author}

\section{Shigetaka Tounou}

Teikyo University Chiba Medical Center - Gastroenterology

3426-3 Anesaki

Ichihara

Chiba 299-0111

Japan

Fax: +81-436-614773

s-tounou@med.teikyo-u.ac.jp
Many guidelines for the management of antithrombotic therapy in endoscopic procedures state that warfarin should be replaced by heparin in high risk endoscopic procedures. However, heparin bridging therapy is costly, requires a long hospital stay, and is indicated as a risk factor for bleeding after endoscopic submucosal dissection (ESD). It is not yet clear whether it is better to perform gastric ESD on continuous warfarin therapy or heparin bridging therapy. We report the case of a 65-year-old Japanese man who had been diag-

\section{Introduction \\ $\nabla$}

Discontinuation of antiplatelet therapy with aspirin may cause stent thrombosis in patients with coronary stents, and discontinuation of anticoagulation therapy with warfarin may cause serious thromboembolic complications in patients after mechanical mitral valve replacement. Endoscopic submucosal dissection (ESD) is an effective treatment for early gastric cancers [1], but management procedures for antiplatelet therapy in ESD have not been established. Guidelines from the European Society of Gastrointestinal Endoscopy [2] recommend discontinuation of all antiplatelet therapy including low dose aspirin in high risk endoscopic procedures, including endoscopic mucosal resection (EMR), ESD, ampullary resection, endoscopic sphincterotomy with large balloon papillary dilation, and fine-needle aspiration biopsy of a cystic lesion using endoscopic ultrasonography. In the Japan Gastroenterological Endoscopy Society guidelines for management of antiplatelet therapy and anticoagulation therapy in endoscopic procedures [3], low dose aspirin and cilostazol can be continued in high risk endoscopic procedures, including ESD. On the other hand, the guidelines published in many countries all state that warfarin should be replaced with heparin (heparin bridging therapy) in high risk endoscopic procedures [2-5]. However, heparin nosed with early gastric cancer. He had a past medical history of metallic valve replacement for mitral valve regurgitation, coronary artery disease with bare metal stent, and coronary artery bypass graft. Warfarin and low dose aspirin had been used to prevent thromboembolic events in the metallic mitral valve and coronary artery stent. We performed gastric ESD safely on continuous warfarin and low dose aspirin without any complications.

bridging therapy is costly and requires long hospital stays, and is indicated as a risk factor for bleeding after ESD [6]. Recently, the safety of pacemaker or defibrillator surgery without interruption of warfarin has been reported [7,8], and continuous warfarin use is considered better than heparin bridging therapy in this type of surgery. However, it has not been clarified whether it is better to perform gastric ESD on continuous warfarin therapy or heparin bridging therapy. Here, we report a case in which we performed gastric ESD on continuous warfarin and low dose aspirin therapy.

\section{Case report \\ $\nabla$}

A 65-year-old Japanese man visited our hospital for treatment of early gastric cancer. He had a history of diabetes mellitus, metallic valve replacement for mitral valve regurgitation, coronary artery disease with bare metal stent, and coronary artery bypass graft. Warfarin and low dose aspirin had been used to prevent thromboembolic events in the metallic mitral valve and coronary artery stent. The international normalized ratio of prothrombin time (PT-INR) had been controlled at around 2.0 (1.5-2.5). A superficial elevated type (0 - Ila) early gastric cancer of $6 \mathrm{~mm}$ in size had been found at another medical facility and it was 

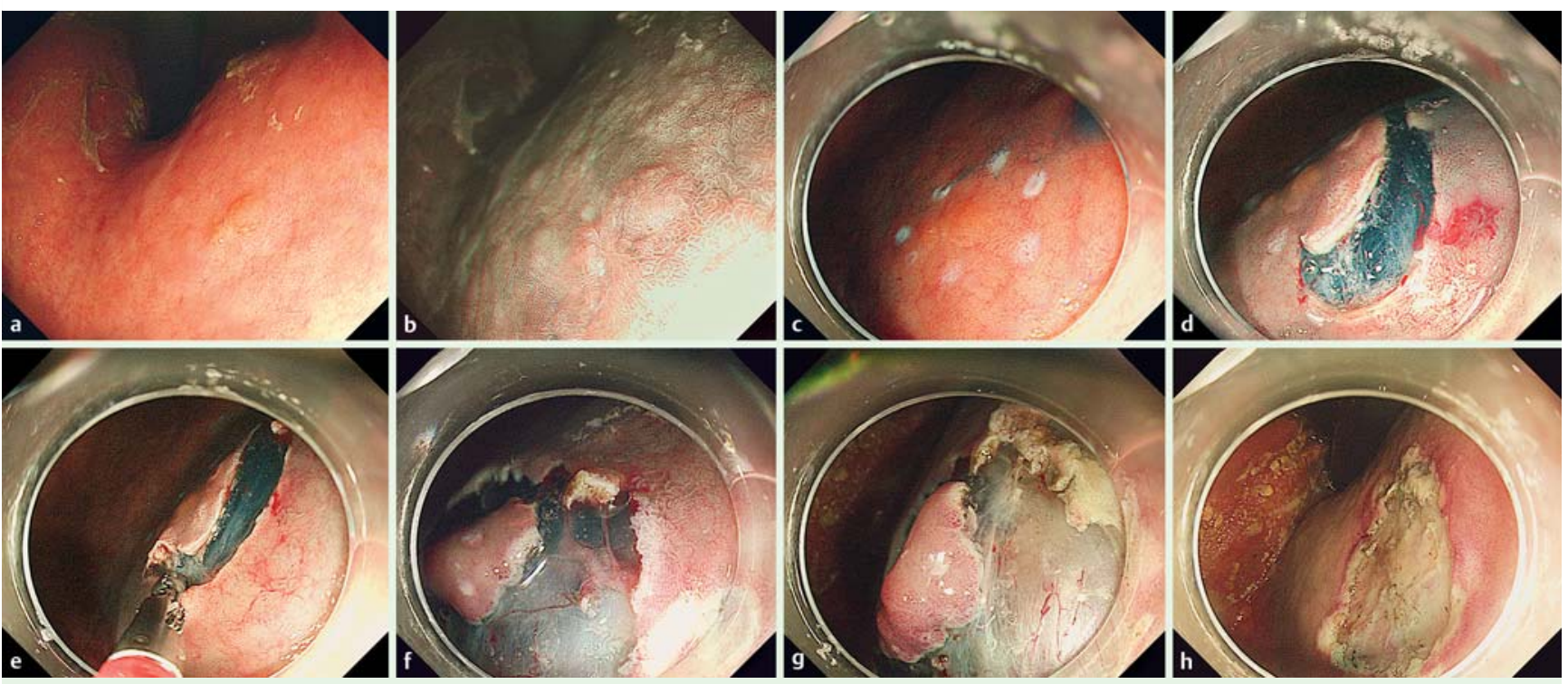

Fig. 1 a Slightly elevated lesion located in the lesser curvature of the upper part of the stomach, and $6 \mathrm{~mm}$ in size, in a 65-year-old man. b High power endoscopic view of the lesion with narrow-band imaging. c Marking the area surrounding the lesion before starting endoscopic submucosal dissection (ESD). d Very shallow mucosal cutting was performed with a Dual knife to avoid injury to vessels in the submucosal layer. e Pre-coagulation with a hot biopsy forceps was carried out before dissecting the submucosal layer. $\mathbf{f}$ Large vessels were observed in the submucosal layer. $\mathbf{g}$ Status of visible vessels in the submucosal layer after coagulation using the hot biopsy forceps. $\mathbf{h}$ Status after hemostasis for the post-ESD ulcer.

located in the lesser curvature of the upper part of the stomach ( $\bullet$ Fig. 1 a, b). The histopathology diagnosis of a biopsy specimen was well-differentiated tubular adenocarcinoma (tub1). Coagulation of the lesion might have been a useful treatment option but the patient desired complete resection and a definite pathological diagnosis. Endoscopic mucosal resection (EMR) was another treatment option but the lesion was small, and the risk of bleeding or perforation was thought to be low. However, we felt that ESD was the ideal therapy for this lesion and the patient also desired it. We consulted the cardiologist who prescribed warfarin and aspirin about interruption of antithrombotic agents, and who strongly recommended their continuous use or heparin bridging therapy and aspirin because the patient had a high thromboembolic risk. We explained to the patient that heparin bridging therapy was recommended in many guidelines but it was not clear whether it was better to perform gastric ESD on continuous warfarin therapy or heparin bridging therapy, and that heparin bridging therapy would need a longer hospital stay and have higher medical costs. We also explained that ESD with continuous warfarin use was a challenging therapy and the risk of post-ESD bleeding was unknown. After our explanation, the patient opted to receive ESD with continuous warfarin and aspirin therapy rather than heparin bridging therapy and informed consent for this therapy was obtained. The PT-INR was 1.67 on the morning of performing ESD, and it was performed on warfarin and aspirin therapy. The procedure was performed by a highly experienced endoscopist using a regular video-endoscope (GIFHQ290; Olympus Medical Systems Co., Tokyo, Japan) with distal attachments (D-201-11804; Olympus Medical Systems). As the ESD device, a Dual knife (KD-650L; Olympus Medical Systems) was used. The electrical generator was a VIO 300D (Erbe Co., Tübingen, Germany) with a setting of endocut effect 3, $120 \mathrm{~W}$ for mucosal incision and forced coagulation effect 3, 50 W for submucosal dissection. Usually, swift coagulation is used in submucosal dissection but we consider that forced coagulation is more effective for preventing hemorrhage during ESD in patients on antithrombotic therapies.

Hemostasis was achieved with a hot biopsy forceps (Radial Jaw 4 , Boston Scientific, United States) with soft coagulation at 70W. At the beginning of ESD, very shallow mucosal cutting was performed with a Dual knife to avoid injury to vessels in the submucosal layer ( $\bullet$ Fig. 1 c, d), and pre-coagulation with the hot biopsy forceps was carried out before dissecting the submucosal layer $(\bullet$ Fig. 1 e,f, g). When there was bleeding during ESD, hemostasis was immediately applied and all visible vessels in the post-ESD ulcer were thoroughly burned out using the hot biopsy forceps after completion of ESD ( $\bullet$ Fig. $\mathbf{1} \mathbf{h}$ ).

Second-look endoscopy was performed on the day following ESD, which showed there was no blood clotting in the stomach ( $\bullet$ Fig. $2 \mathrm{a}$ ). There was no active bleeding and there were no apparent vessels in the post-ESD ulcer, but preventive hemostasis with the hot biopsy forceps was applied to the reddish area mainly in the margin of the post-ESD ulcer.

The patient was permitted to drink water 2 hours after ESD and after the second-look endoscopy, a liquid diet was started in the evening of the day that it was performed. On the day of ESD and on the following day, $10 \mathrm{mg}$ of iv omeprazole twice a day was administered. Oral rabeprazole at $10 \mathrm{mg}$ twice daily was started 2 days after ESD and continued for 30 days. Hemoglobin levels, platelet counts, and PT-INR were checked on the morning after ESD and 7 days after ESD ( Table 1). PT-INR values were 1.68 and 1.73 on the morning after ESD and 7 days after ESD, respectively. The patient was hospitalized for 7 days in accordance with the clinical guidelines of our hospital and checked for signs of postESD bleeding. Third-look endoscopy was performed 7 days after ESD to confirm the hemostatic status of the post-ESD ulcer $(\bullet$ Fig. 2 b). At discharge, signs of gastric bleeding were carefully explained to the patient and he was asked to visit hospital immediately when he noticed any of them. The patient visited our outpatient clinic 30 days after ESD for follow-up after ESD. No signs 

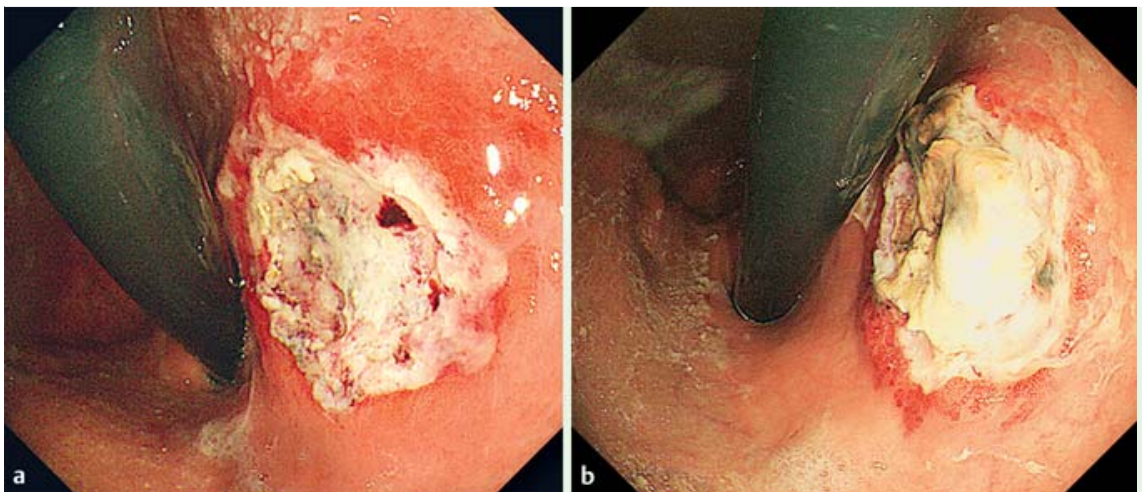

Fig. 2 a Second-look endoscopic view of the postESD ulcer on the day following ESD. There was no active bleeding and there were no apparent vessels in the post-ESD ulcer. $\mathbf{b}$ Third-look endoscopic view of the post-ESD ulcer 7 days after ESD.

Table 1 International normalized ratio of prothrombin time (PT-INR), hemoglobin levels and platelet cell counts associated with endoscopic submucosal dissection (ESD).

\begin{tabular}{|llllll|}
\hline & \multicolumn{6}{c|}{ Days before or after ESD } \\
\hline & $\mathbf{- 1 4}$ & $\mathbf{0}$ & $\mathbf{1}$ & $\mathbf{7}$ & $\mathbf{3 0}$ \\
\hline PT-INR & 1.78 & 1.67 & 1.68 & 1.73 & 2.08 \\
\hline Hemoglobin $(\mathrm{g} / \mathrm{L})$ & 121 & 121 & 119 & 117 & 123 \\
\hline Platelet cell counts $\left(\times 10^{9} / \mathrm{L}\right)$ & 205 & 204 & 177 & 190 & 217 \\
\hline
\end{tabular}

PT-INR, international normalized ratio of prothrombin time; ESD, endoscopic submucosal dissection.

of gastric bleeding were noticed and no drop in hemoglobin was seen $(\bullet$ Table 1$)$.

The resected specimen measured $12 \times 10 \mathrm{~mm}$ and the early gastric cancer measured $5 \times 3 \mathrm{~mm}(\bullet \mathbf{F i g} . \mathbf{3 a}, \mathbf{b})$. Unfortunately, a small mucosal slit occurred in the resected specimen during its retrieval but this did not affect the pathological examination $(\odot$ Fig. 3c). Histopathology revealed a well-differentiated adenocarcinoma of $4 \mathrm{~mm}$ in diameter located within the mucosal layer, with no evidence of lymphovascular infiltration. The vertical and horizontal margins were free of carcinoma ( $\bullet$ Fig. 4).

\section{Discussion}

$\nabla$

ESD is now considered to be an effective and safe treatment for early gastric lesions (early gastric cancers and adenomas), but the perioperative management of antithrombotic therapies is still controversial. Continuous aspirin use in ESD has been considered to be safe in several previous studies $[9,10]$, but the effect of continuous warfarin use has not been investigated. Also, many guidelines for endoscopic procedures [2-5] state that warfarin therapy should be replaced with heparin bridging therapy, but the safety of heparin bridging therapy has not been confirmed [6]. In studies comparing continuous oral anticoagulation therapy with heparin bridging therapy in surgery for implantation of cardiac rhythm devices, continuous oral anticoagulation therapy was better than heparin bridging therapy because there was less post-operative bleeding and hospital stays were shorter [7, 8]. We are not aware of any speculated reason for more bleeding in patients with heparin bridging therapy. However, there will be a need to administer warfarin concomitantly with heparin during bridging therapy after a surgical or endoscopic procedure, and the anticoagulation action of both heparin and warfarin will be combined before conclusion of the therapy. Thus, the anticoagulation action will be much greater than for warfarin monotherapy. This might be a reason for greater bleeding risk in patients with heparin bridging therapy.

The present case shows that ESD can be performed safely without interrupting warfarin with aspirin therapy, and it would be beneficial for patients on antithrombotic therapies. However, in such
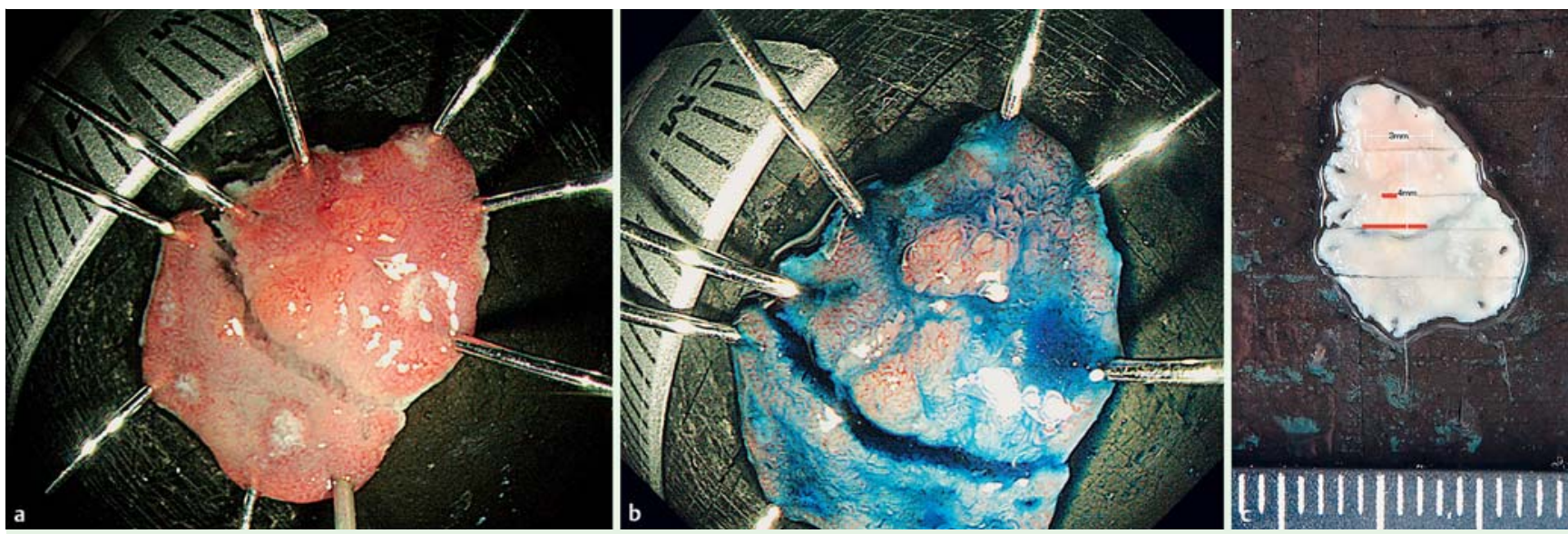

Fig. 3 a View of resected specimen. Resected specimen measured $12 \times 10 \mathrm{~mm}$ and early gastric cancer measured $5 \times 3 \mathrm{~mm}$. A small mucosal slit occurred in the resected specimen during retrieval, but this did not affect the pathological examination. b View of resected specimen with Indigo Carmine solution. c Mucosal cancer area in resected specimen indicated by red lines. 


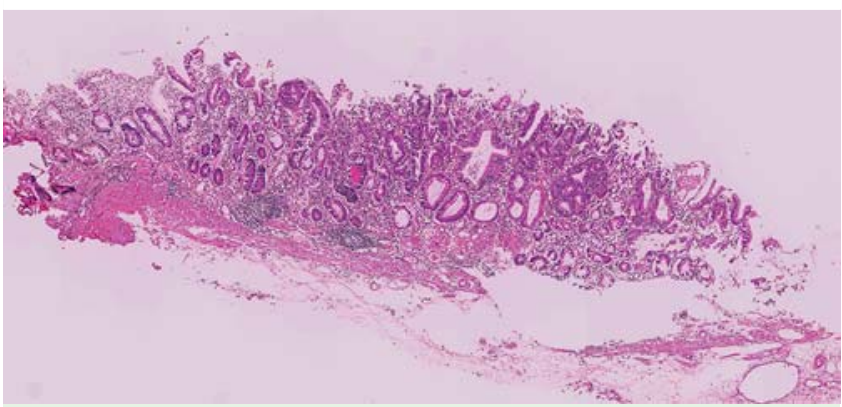

Fig. 4 Final diagnosis was intramucosal gastric cancer of $4 \mathrm{~mm}$ in diameter, a well differentiated adenocarcinoma with no lymphovascular infiltration $(\mathrm{H} \& \mathrm{E}, \times 40)$.

cases, care is required in preventive hemostasis during the ESD procedure as well as in endoscopic follow-up after ESD.

The following are important points in performing ESD safely in patients on antithrombotic therapies: (1) at the beginning of ESD, only the mucosal layer should be cut to avoid injury to vessels in the submucosal layer; (2) before starting dissection of the submucosal layer, visible vessels in it should be pre-coagulated with hot biopsy forceps or other hemostatic devices; (3) during dissection of the submucosal layer, immediate hemostasis should be applied when hemorrhage is seen; (4) after completing ESD, all of the visible vessels in the post-ESD ulceration should be burned out with hot biopsy forceps or other hemostatic devices; and (5) to prevent late onset hemorrhage, second-look endoscopy should be performed on the day following ESD, and thirdlook endoscopy 1 week after ESD. Although the effectiveness of second-look and third-look endoscopy after ESD is unclear, we feel that additional preventive hemostasis at second-look and third-look endoscopy might be effective in preventing post-ESD bleeding in patients on antithrombotic therapies.

This is the first published case of gastric ESD in a patient on continuous warfarin and aspirin therapy. However, the gastric lesion was very small and PT-INR was not particularly high. When performing gastric ESD for larger gastric lesions with stronger anticoagulation therapies, greater care would be essential in the endoscopic procedure and follow-up because gastric lesion size is thought to be a risk factor for post-ESD bleeding.

\section{Competing interests: None}

\section{References}

1 Oda I, Saito D, Tada $M$ et al. A multicenter retrospective study of endoscopic resection for early gastric cancer. Gastric Cancer 2006; 9: $262-$ 267

2 Boustière C, Veitch A, Vanbiervliet $G$ et al. Endoscopy and antiplatelet agents. European Society of Gastrointestinal Endoscopy (ESGE) Guideline. . Endoscopy 2011; 43: $445-458$

3 Fujimoto K, Fujishiro M, Kato M et al. Guidelines for gastroenterological endoscopy in patients undergoing antithrombotic treatment. Dig Endosc 2014; 26: $1-14$

4 Anderson MA, Ben-Menachem T, Gan SI et al. Management of antithrombotic agents for endoscopic procedures. Gastrointest Endosc 2009; 70: 1060 - 1070

5 Veitch AM, Barglin TP, Gershlich AH et al. Guidelines for the management of anticoagulant and antiplatelet therapy in patients undergoing endoscopic procedures. Gut 2008; 57: 1322 - 1329

6 Yoshio T, Nishida T, Kawai $N$ et al. Gastric ESD under heparin replacement at high-risk patients of thromboembolism is technically feasible but has a high risk of delayed bleeding: Osaka University ESD Study Group. Gastroenterol Res Pract 2013; 2013: 365 -830

7 Feng $L, L i Y$, Li J et al. Oral anticoagulation continuation compared with heparin bridging therapy among high risk patients undergoing implantation of cardiac rhythm devices. Thromb Haemost 2012; 108: $1124-1131$

8 Bienie DH, Healey JS, Wells GA et al. Pacemaker or defibrillator surgery without interruption of anticoagulation. NEJM 2013; 368: 2084-2093

$9 \mathrm{Lim} \mathrm{JH}$, Kim SG, Kim JW et al. Do antiplatelets increase the risk of bleeding after endoscopic submucosal dissection of gastric neoplasms? Gastrointest Endosc 2012; 75: 719-727

10 Tounou S, Morita Y, Hosono T. Continuous aspirin use dose not increase post-endoscopic submucosal dissection bleeding risk for gastric neoplasms in patients on antiplatelet therapy. EIO 2015; 03: E31-E38 